\title{
Clinical heterogeneity of a population of patients admitted to the Emergency Department with a diagnosis of COPD-exacerbation: Relevance of cardiovascular comorbidities
}

\author{
Paolo Groff, ${ }^{1}$ Giuseppina Petrelli, ${ }^{2}$ Paolo Giorgini, ${ }^{2}$ Roberto Pilotti, ${ }^{2}$ Vito Maurizio Parato, ${ }^{3}$ \\ Andrea Fabbri ${ }^{4}$ \\ ${ }^{1}$ Emergency Department, Santa Maria della Misericordia Hospital, Perugia; ${ }^{2}$ Emergency Department, \\ Madonna del Soccorso Hospital, San Benedetto del Tronto; ${ }^{3}$ Cardiology Unit, Madonna del Soccorso \\ Hospital, San Benedetto del Tronto; ${ }^{4}$ Emergency Department, Morgagni-Pierantoni Hospital, Forlì, Italy
}

\begin{abstract}
FEV1-based Chronic Obstructive Pulmonary Disease (COPD) severity does not account for the complexity of the disease. Recent studies point to the high frequency of comorbidities responsible for unfavorable outcomes. There is a lack of data on this concerning the patient evaluated in the emergency setting. Aim of the study was to prospectively evaluate patients admitted to the ED for "exacerbated COPD" to describe their clinical heterogeneity and the influence that it may have on outcomes: death, length of hospitalization, exacerbation recurrence. The following data were recorded: history, symptoms, blood gas analysis, laboratory and
\end{abstract}

\footnotetext{
Correspondence:Paolo Groff, Emergency Department, Santa Maria della Misericordia Hospital, Piazzale Menghini 1, 06129 Perugia, Italy. Tel.: +393336820921.

E-mail: Paolo.groff@ospedale.perugia.it

Key words: COPD exacerbation; COPD phenotypes; cardiovascular comorbidities; prognosis; emergency department.

Conflicts of interest: No one. This work was not supported by any grant.

Availability of data and materials: All data generated or analyzed during this study are included in this published article.

Ethics approval and consent to participate: All procedures performed in studies involving human participants were in accordance with the ethical standards of the Institutional Research Committee and with the 1964 Helsinki declaration and its latest amendment.

Informed consent: Informed consent was obtained from a legally authorized representative(s) for anonymized patient information to be published in this article.

Received for publication: 13 November 2020 .

Revision received: 3 February 2021.

Accepted for publication: 3 February 2021

This work is licensed under a Creative Commons Attribution 4.0 License (by-nc 4.0).

${ }^{\circ}$ Copyright: the Author(s), 2021

Licensee PAGEPress, Italy

Emergency Care Journal 2021; 17:9502

doi:10.4081/ecj.2021.9502
}

radiological findings and comorbidities. Each patient underwent electrocardiography, echocardiography and spirometry. In order to identify a correlation between these variables and the selected outcomes, a multivariate linear logistic regression analysis was carried out. This study was conducted on 41 eligible patients consecutively admitted to the emergency room for exacerbated COPD. A consistent proportion showed ECG, Echocardiographic and laboratory abnormalities. At spirometry a FEV $1<30 \%$ of predicted was detected in $37 \%$ of patients. Cardiovascular comorbidities came out to be very frequent (hypertension, heart failure and coronary artery disease in particular). The history of heart failure was related to the risk of re-hospitalization within three months, while pneumonia, a low $\mathrm{pH}$ and a low FEV1 predicted a hospital stay $>7$ days. Our study shows that the term "exacerbated COPD" underscores a heterogeneous population, with a high prevalence of cardiovascular comorbidities, which significantly influence outcomes. Multicenter studies are needed to better investigate the clinical relevance of these findings.

\section{Introduction}

The importance of comorbidities in Chronic Obstructive Pulmonary Disease (COPD) has been widely reiterated in recent editions of the GOLD document. ${ }^{1}$ In addition, in the various formulations of the so-called phenotypes of this disease, the entity indicated as "bronchitis with co-morbidities"2,3 takes on particular importance; the latter is characterized by a high frequency of exacerbations requiring hospitalization and a low life expectancy. ${ }^{4-}$ ${ }^{6}$ Although exacerbations of the disease, and in particular those requiring hospitalization, represent adverse events of great importance in its natural history, especially in relation to prognosis, ${ }^{7,8}$ most of the information relating to the problem of comorbidities and their role derives from the large studies carried out on outpatient or hospitalized patients in stable conditions. ${ }^{2,4}$

In particular, there is a lack of data on the clinical heterogeneity profile of patients in the Emergency Department diagnosed with exacerbated COPD and its potential role in influencing their management and short and medium-term prognosis. It is in fact conceivable that patients characterized by different clinical features present, in the acute phase, different management problems and that these problems may, at least in part, be supported by a differential composition of comorbidities. It is also possible that the identification of particular management issues, such as the need for a prolonged period of stay in hospital or a particular risk in terms of survival in hospital or shortly after discharge suggest a different monitoring program for these 
patients, and therefore help to rationalize the allocation of resources. Finally, greater awareness of the set of coexisting pathologies in the exacerbated patient may allow a new approach to medical therapy, where the evidence suggests the opportunity to address all the different comorbidities by following the respective guidelines for each. ${ }^{9}$

The purpose of this study was, therefore, to prospectively study all patients referred to the emergency room of the "Madonna del Soccorso" Hospital in San Benedetto del Tronto and subsequently admitted to the Intensive Short Observation area (ISO) of the same operating unit with a diagnosis of "exacerbated COPD" for a period of one year starting from 13.01.2016. The particular subject of this survey was: i) the description of the clinical heterogeneity of the population of patients who access the Emergency Department with a diagnosis of "exacerbated COPD"; ii) the influence that some epidemiological characteristics, comorbidities, laboratory or instrumental findings may have in influencing certain outcomes such as death during or after hospitalization, the length of stay in hospital, the number of exacerbations within three months after the index event or in the subsequent period.

\section{Materials and Methods}

This study was conducted at the Emergency Department (ED) of the "Madonna del Soccorso" Hospital of San Benedetto del Tronto in the Area Vasta n. 5 of the Single Regional Health Authority of the Marche Region. The aforementioned community hospital serves a catchment area of 100,000 inhabitants with a number of accesses to the emergency room for the year 2016 equal to 38,706 . The ED is organized in an Emergency Room (ER) where patients are accepted and first stabilized and in an inpatient area ("Medicna d'Urgenza") where the subsequent treatment takes place in the form of Intensive Short Observation (ISO, usually within 48 hours of entry) or ordinary hospitalization (usually within 72 hours of entry). Patients who, at the end of the treatment period in the ED inpatient area, need to continue the therapeutic process are transferred to other departments of the hospital. During the year 2016, 2,844 patients were admitted for observation at the OBI of the ED; of these, about half continued the process in other departments; 82 patients, after the observation period at OBI, continued the process as an ordinary hospitalization in the same unit. For the purposes of this study, all patients admitted to the OBI after evaluation in the ER with an initial diagnosis of "exacerbated COPD" were considered eligible for enrollment, if they were able to express informed consent to be included, were able to correctly perform a spirometry after clinical stabilization, and had performed a blood sample (including leukocyte count, $\mathrm{C}$ reactive Protein - CRP - and NT fragment of the Atrial Natriuretic Peptide -NT-proBNP), an arterial blood gas analysis with expression of FiO2 (fraction of inspired oxygen), an electrocardiogram (ECG) and an imaging exam of the chest (conventional X-ray or chest Computed Tomography-CT). Since in a certain number of patients the emergency room doctor had autonomously performed a high sensitivity troponin determination, not foreseen by the study protocol, the researchers decided, at a later time, to acquire and process this data for those patients for whom it was available.

Both in the Emergency Room and in the subsequent phase in the OBI, the physician in charge of the patient was free to choose the medical treatment to be applied; all patients included in the study underwent antibiotic therapy, intravenous steroid therapy
(Methylprednisolone 40-60 $\mathrm{mg} /$ day); bronchodilation by nebulization of salbutamol (5000 mcg x 4/day).

During their stay in the ISO, all patients underwent a complete echocardiographic study carried out by a consultant cardiologist expert in the method and unaware of the purpose of the study using a Vivid E9 device (General Electric Healtcare, Buckingamshire, UK). In conditions of clinical stabilization, that is when the physician in charge believed that the general conditions and the level of dyspnea allowed it, all patients underwent simple spirometry (slow vital capacity and forced vital capacity) using a turbine spirometer (MicroQuarck USB Spirometer, linked to Omnia software, Guangzhou, China). This test had the purpose to better define the presence of an obstructive derangement of the airways, thus confirming the clinical diagnosis performed by the ER doctor, and to assess its severity after the medical therapy had been administered and the acute exacerbation stabilized, thus allowing to evaluate the severity of the underlying disease. Previously published methods were used to perform the spirometric test. ${ }^{10-12}$

For each patient included in the study, a computerized data collection form was compiled including the following items: age; sex; COPD exacerbation symptoms upon entry (presence / absence of dyspnea, cough, changes in sputum or fever); history of COPD (diagnosis of COPD pre-existing at the current ED visit: present or absent); history of smoking (present or absent); availability of a previous spirometric test (present / absent); ongoing treatment for COPD prior to the current access (present / absent); history of coronary ischemic disease (diagnosis of CAD prior to the current access: present or absent); history of heart failure (diagnosis of heart failure prior to the current visit at the emergency room: present or absent); current presence/absence of atrial fibrillation arrhythmia; presence/absence of obesity (BMI $>30)$; presence/absence of diabetic disease; presence/absence of previously diagnosed arterial hypertension; presence/absence of chronic renal failure; presence/absence of anemia; presence/absence of pneumonia. Among the laboratory parameters detected at the time of entry into the $\mathrm{ED}$, the following were recorded: arterial blood gas analysis: $\mathrm{PaO}_{2} ; \mathrm{PaO}_{2} / \mathrm{FiO}_{2} ; \mathrm{PaCO}_{2}$; $\mathrm{HCO}_{3}^{-} ; \mathrm{pH}$; white blood cell count; C-reactive protein; NT-proBNP; troponin $\mathrm{T}$ (limited to patients in whom this test was performed). Among the instrumental parameters, the presence/absence of electrocardiographic anomalies (of frequency, rhythm, impulse conduction, or the morphology of the complexes) and the presence/absence of an imaging of the chest compatible with emphysema, bronchitis or pulmonary congestion was detected. These items were entered on the basis of the judgment of a cardiologist consultant and a radiologist both unaware of the purpose of the study. The following parameters were derived from the echocardiographic report: ejection fraction (\%) of the Left Ventricle (LV); presence/absence of LV wall dyskinesis; presence/absence of LV diastolic dysfunction; PAPs ( $\mathrm{mmHg}$ ). The following spirometric parameters were also entered: presence/absence of obstructive disturbance and FEV1 (Forced Expired Volume in the first second) value (\% predicted). Finally, for each patient, the following outcomes were collected: presence/absence of a new exacerbation within three months of the index event; presence/absence of a new exacerbation between the index event and the date of 15/05/2017 in which data processing began (total time of 17 months); length of stay in hospital; presence/absence of death in the period between the index event and the date of $15 / 05 / 2017$. The data relating to subsequent hospitalizations for "COPD exacerbation" were obtained by consulting the computerized archive of patients who accessed the 
ED; the data relating to the length of hospitalization were obtained by consulting this same archive and the general archive of the hospital's medical records; the data relating to any death were obtained by consulting the Epidemiological Office of our Health Authority.

The study was approved by the Hospital Management Board; all patients expressed informed consent to participate; sensitive patient data have been treated with full respect for privacy; the opinion of the Ethics Committee was waived due to the observational, non-interventional nature of the study and the fact that no patient received treatments and procedures outside of what falls within the principles of best medical practice.

The reported data were used for an epidemiological description of the population of patients referred to our Emergency Department with a diagnosis of "exacerbated COPD". In order to identify the potential relationship between epidemiological factors, the presence of comorbidities, laboratory and instrumental data and the selected outcomes, an analysis was conducted using linear logistic regression.

\section{Statistic analysis}

Mean value, standard deviation, median, interquartile range, number of cases, percent with $95 \%$ confidence interval were used to describe data distribution. Fisher's exact test for categorical variables and Student t-test for continuous variables were used to compare variables between groups. The following variables were tested for multivariable analysis: sex, age $>75$ years, $\mathrm{pH}<7.35$, $\mathrm{PaO}_{2} / \mathrm{FiO}_{2}<200$, NT-proBNP $>1500$ pg/ml, Left Ventricle Ejection Fraction $<55 \%$, PAPs $>35 \mathrm{mmHg}$, FEV $1<30 \%$ predicted, presence of an history of Coronary Artery Disease, Heart Failure, Atrial Fibrillation, Obesity, Diabetes, Hypertension, Chronic Renal Failure, anemia, pneumonia at admission. A multivariable model was developed by stepwise forward analysis of factors considered significant in univariable analysis and according to clinically relevant predictors. For the model building, the variables were selected on the basis of previous reports and a putative association with main outcome measures (death throughout the study period; recurrence of exacerbation within three months from the index admission; recurrence of exacerbation throughout the entire study period; length of hospital stay $>7$ days). As it is recommended that covariates should be introduced generously into the model, we included a large number of covariates independently of significance thresholds or other selection criteria. The colinearity of combination of variables was tested by the variation inflation factor $(<2$, not significant). The Statistical Package for the Social Science SPSS/PC+ (20.0 edition) was used in statistical analyses.

\section{Results}

In the period between 13.01.2016 and 09.02.2017 119 patients were held for observation at the Emergency Medicine with a diagnosis of "exacerbated COPD". Among these, 33 (28\%) were unable to perform spirometry correctly due to various causes (inability to collaborate due to cognitive deterioration: 12; excessive air losses due to edentulism or ischemic cerebral outcomes: 8; persistence of excessive dyspnea in the second-third day: 11; transfer to another department before the possibility of taking the exam: 10). In 20 cases (17\%) spirometry was not performed due to the need to continue treatment with non-invasive ventilation; 17 (14\%) patients were not enrolled due to incomplete compilation of the data collection form and $8(6.7 \%)$ for reasons that could not be specified (Figure 1).
Forty-one patients $(34.4 \%)$ were therefore available for the study. Among these, there were 25 males (61\%); the average age was 75.9 (9.1) years. All patients presented an increase in dyspnea as a guiding symptom for access to the ED $(41,100 \%)$. Thirty-five patients $(85.3 \%)$ reported a previous diagnosis of COPD, although only $13(31.7 \%)$ had a previous spirometry and $15(36.5 \%)$ could not report on it. A history of cigarette smoking was detectable in 27 patients $(65.8 \%)$; in four patients $(9.7 \%)$ this data was not detected. only 24 patients $(58.5 \%)$ reported taking a specific therapy for COPD at home. Epidemiological data are summarized in Table 1.

With respect to the detectable comorbidities, 9 patients $(21.9 \%)$ reported a previous diagnosis of coronary heart disease, while in 13 cases $(31.7 \%)$ there was a history of heart failure. 10 patients $(24.3 \%)$ presented with atrial fibrillation arrhythmia, while $31(75.6 \%)$ were hypertensive. Finally, 11 patients $(26.8 \%)$ were diagnosed with a bronchopneumonic consolidation at the time of evaluation in the ER. It should be noted that 32 patients (78\%) had more than one comorbidity, while only $7(17 \%)$ had only one comorbidity and $2(4.8 \%)$ had no comorbidity. For all comorbidities see Table 2

As to blood gas indices, the average $\mathrm{PaO} 2$ in the entire group of patients was 56.31 (13.9), the mean $\mathrm{pH}$ was 7.38 (0.071), with a value lower than 7.35 in 12 patients $(29.2 \%)$.

As to the laboratory indices, the mean CRP value was 4.48 (5.9) $\mathrm{mg} / \mathrm{dL}$ with values greater than $0.5 \mathrm{mg} / \mathrm{dL}$ in 36 patients (87.8\%); high sensitivity troponin $\mathrm{T}$ was available only for 14 patients $(34.1 \%)$ and had a mean value of $34.8(20.3) \mathrm{pg} / \mathrm{mL}$ with values greater than $15 \mathrm{pg} / \mathrm{mL}$ in 12 of them $(85,7 \%)$; NT-pro-BNP was available in 36 patients $(87.8 \%)$ and averaged 6654 (10813) $\mathrm{pg} / \mathrm{mL}$, and in any case higher than $1800 \mathrm{pg} / \mathrm{mL}$ in 18 patients $(50 \%)$.

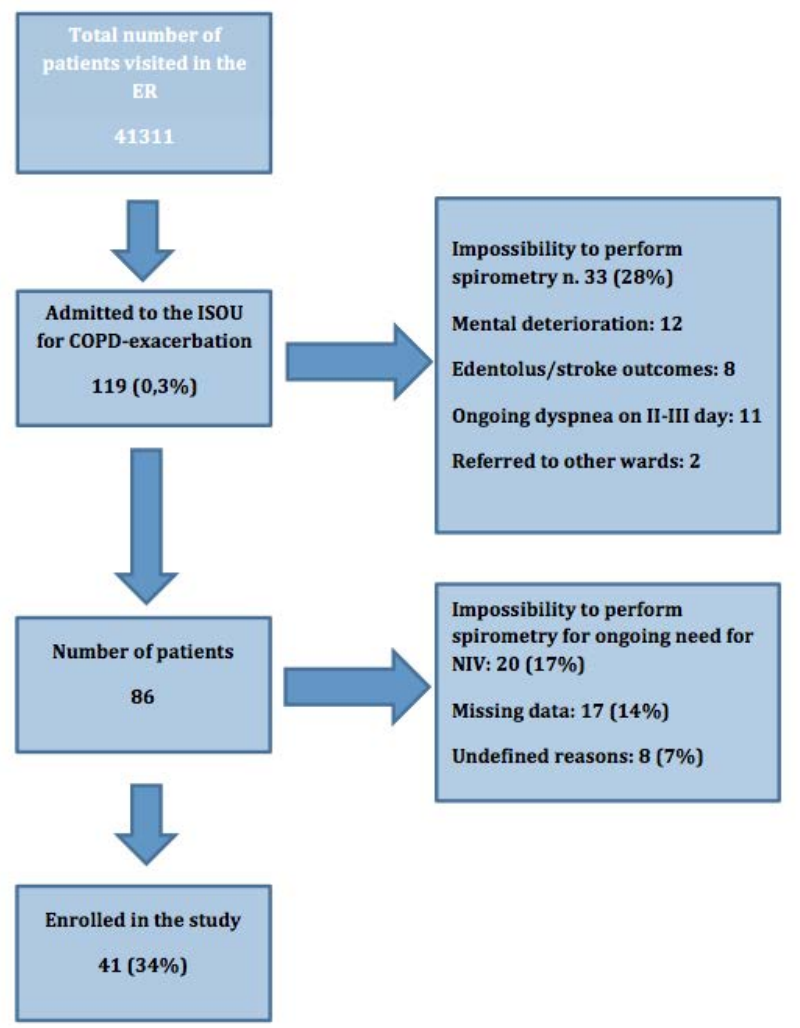

Figure 1. Patients selection process. 
Table 1. Epidemiological data.

\begin{tabular}{|c|c|c|c|c|c|}
\hline \multicolumn{6}{|l|}{ Age and sex } \\
\hline Males & Females & & Mean Age (SD) & Lower limit & Upper limit \\
\hline 25 & 16 & & $75,95(9,17)$ & 58 & 95 \\
\hline \multicolumn{6}{|c|}{$\begin{array}{l}\text { Guiding symptoms at admission } \\
\text { Symptom }\end{array}$} \\
\hline Dyspnoea & & 41 & & 100 & \\
\hline Cough & & 22 & & 53,65 & \\
\hline Increased/modified sputum & & 13 & & 31,70 & \\
\hline Fever & & 20 & & 48,78 & \\
\hline More than one symptom & & 29 & & 70,73 & \\
\hline \multicolumn{6}{|l|}{ COPD status } \\
\hline Hystory & n. & & $\%$ & Not available & $\%$ \\
\hline COPD & 35 & & 85,36 & 0 & 0 \\
\hline Smoke & 27 & & 65,85 & 4 & 9,75 \\
\hline Spirometry & 13 & & 31,70 & 15 & 36,58 \\
\hline Therapy & 24 & & 58,53 & 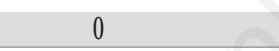 & 0 \\
\hline
\end{tabular}

Chest imaging was judged compatible with "bronchitis" in 19 patients $(46.3 \%)$, congestive in 14 patients $(34.1 \%)$, emphysematous in 7 patients $(17 \%)$ and normal in 1 patient only $(2,4 \%)$. The cardiologist found electrocardiographic anomalies, as defined in the previous paragraph, in 31 cases $(75.6 \%)$, while at the echocardiographic examination the mean value of the ejection fraction of the left ventricle was $56.5(9.1) \%$, with values below $55 \%$ in 8 cases $(19.5 \%)$. Systolic pulmonary pressure (PAPs) was evaluable in 37 patients $(90.2 \%)$ and had a mean value of 43 (12) $\mathrm{mmHg}$, resulting in more than $35 \mathrm{mmHg}$ in 30 of them $(73 \%)$. The ventricular filling pattern was evaluable in 31 patients $(75.6 \%)$, with anomalies detected in 17 cases $(54.8 \%)$, while $7 / 41$ patients $(17 \%)$ were carriers of alterations in the wall kinetics.

Finally, spirometry allowed the detection of an obstructive functional deficit in 34 patients $(82.9 \%)$ and, interestingly, nonobstructive in $7(17 \%)$. The mean FEV1 value was $34.24(12) \%$ of predicted, but $15 / 41$ patients $(36.5 \%$ ) had a value below $30 \%$. (for the instrumental investigations see Table 3 ).

Patients included in the study had a mean length of hospital stay of 7.2 (6.7) days; in 14 cases it lasted more than 7 days $(34.1 \%)$.

Nine patients $(21.9 \%)$ were re-hospitalized for "COPD exacerbation" within three months of the index event, while, considering the period between the start of the study and the start of data processing (13.01.2016-15.05.2017) this event involved 14 cases $(34.1 \%)$. In this same period 11 patients $(26.8 \%)$ died (for outcomes see Table 4).

Given the small number of patients considered, the multivariate analysis did not allow to associate the risk of death with any of the demographic, clinical, laboratory or instrumental variables considered; on the contrary, the presence of a history of heart failure was significantly associated with the risk of new hospitalization within three months for COPD exacerbation (OR 7.14; 95\% CI 1.4-36; $\mathrm{p}<0.017$ ). Similarly, a hospital stay longer than seven days was significantly associated with the presence of pneumonia (OR 18.28; 95\% CI; $<<0.008$ ); a low $\mathrm{pH}$ value at admission (OR 10.09; 95\% CI 1.18-86.44; p<0.035); a measured FEV1 value of less than $30 \%$ of predicted (OR 6.23; $95 \%$ CI 0.98 $39.5 ; \mathrm{p}<0.052$ ). No significant association were observed between any of the variables considered and the risk of new hospitalization
Table 2. Detected comorbidities.

\begin{tabular}{lcc} 
Comorbidity & n & $\%$ \\
Hypertension & 31 & 75,60 \\
Heart Failure & 13 & 31,70 \\
\hline Anemia & 13 & 31,70 \\
Pneumonia & 11 & 26,82 \\
\hline Diabetes & 11 & 26,82 \\
Atrial fibrillation & 10 & 24,39 \\
\hline Coronary heart disease & 9 & 21,95 \\
Chronic renal failure & 9 & 21,95 \\
\hline Obesity & 5 & 12,19 \\
More than one comorbidity & 32 & 78,04 \\
\hline Only one comorbidity & 7 & 17,07 \\
No comorbidities & 2 & 4,87 \\
\hline
\end{tabular}

for exacerbated COPD throughout the longer study period (13.01.2016-15.05.2017).

Table 5 summarizes the association of the individual variables with the outcomes.

\section{Discussion}

Although numerically limited, our patient population largely mirrors what is reported in the literature. ${ }^{1}$ There is a slight prevalence of males, the average age is advanced. The guiding symptoms that led patients to the emergency room are dyspnea, cough, changes in sputum and fever (Table 1), which classically define a clinical COPD exacerbation as persistent worsening of the patient's condition that goes beyond the normal daily variability of the symptoms of disease, has acute onset and forces the patient to undergo medical attention. ${ }^{13}$ Moreover, the diagnosis of "COPD exacerbation" is placed by the Emergency Physician almost exclusively on a clinical basis, that is based on the history of symptoms and their acute variation, given that only a minority of 
patients provide the data of previous spirometries, which are essential to establish the existence of an underlying obstructive disorder. In our series, although 35 patients reported a previous diagnosis of COPD, only 13 exhibited previous spirometry, 15 were unsure whether they had performed the aforementioned examination in the past and all others, including those of the total 41 patients who did not have a previous diagnosis of COPD, were sure they had never done a spirometry. Furthermore, there is a certain incongruity with respect to the home use of specific therapy, given that, in our series, $58.5 \%$ of patients took inhaled bronchodilators which implies, on the one hand, that a certain number of patients receive the prescription of the aforementioned drugs in the absence of a preliminary spirometric assessment, on the other hand the fact that the prescription of the necessary drugs is lower than the real need in the population ( $82.9 \%$ in our series). These data are in line with other studies that tend to demonstrate a low awareness of chronic obstructive pulmonary disease in the population and a general situation of underestimation and undertreatment of the problem. ${ }^{14,15}$

It is also interesting to note that the purely "clinical" diagnosis of the Emergency Physician was confirmed spirometrically in $82.9 \%$ of our cases, as will be discussed later, indicating that a correct anamnestic evaluation of the symptoms, together with the aid of the clinical examination and instrumental and laboratory tests used in this setting allow for an acceptable approximation in making a "presumptive" diagnosis of exacerbated COPD.

Although chronic obstructive disease is largely related to cigarette smoking in the Western world, only $65.8 \%$ of our patients reported being smokers or having smoked; this is in line with the most recent reports which show that a minority, but constantly increasing in percentage, of cases of the disease cannot be correlated with smoking. ${ }^{1}$
Our study confirms what has been widely published in recent times about the importance of comorbidities in the natural history of chronic obstructive pulmonary disease and its exacerbations. ${ }^{16,17}$

In our series 9 patients $(21.9 \%)$ reported a previous diagnosis of coronary heart disease, while in 13 cases $(31.7 \%)$ there was a history of heart failure. Ten patients $(24.3 \%)$ presented with atrial fibrillation, while $31(75.6 \%)$ were hypertensive. Only 5 patients $(12.1 \%)$ had a BMI compatible with obesity, but $11(26.8 \%)$ were carriers of diabetic disease. Nine patients $(21.9 \%)$ were carriers of chronic renal failure and $13(31.7 \%)$ were anemic (Table 2). It should also be remembered that in 11 patients a bronchopneumonic consolidation was present at the entrance to the $\mathrm{ED}$, although in this case it is more correct to speak of an infectious cause of the exacerbation, rather than a real comorbidity. Even in laboratory and instrumental evaluations, high sensitivity troponin $\mathrm{T}$, although available in a limited number of patients, had an altered mean value ( $34.8 \mathrm{pg} / \mathrm{mL})$ and NT-pro-BNP had a mean value of $6654(\mathrm{pg} / \mathrm{mL})$ and was in any case higher than the value of $1800 \mathrm{pg} / \mathrm{mL}$ in $50 \%$ of patients. In addition, on echocardiographic examination, PAPs was compatible with right ventricular engagement in more than $70 \%$ of cases, and an overall non-negligible number of patients had a reduction in the ejection fraction, alterations in the ventricular filling phase or the wall kinetics. Finally, chest imaging was found to be compatible with congestive heart failure in more than a third of cases and almost all patients presented with electrocardiographic changes (Table 3).

Although the research has addressed the problem of comorbidities in a broad way, including nutritional disorders and bone metabolism, systemic metabolic disorders and sleep-related problems, the above data can only highlight the importance of cardiovascular comorbidities especially in the organizational context of the ED. Numerous studies indicate that several overlaps

Table 3. Laboratory and instrumental findings.

\begin{tabular}{|c|c|c|c|c|c|c|c|}
\hline \multicolumn{8}{|c|}{ Laboratory findings } \\
\hline Test & \multicolumn{2}{|c|}{ Mean (SD) } & \multicolumn{5}{|c|}{ Missing data } \\
\hline $\mathrm{WBC}\left(\mathrm{n} / \mathrm{mm}^{3}\right)$ & \multicolumn{2}{|c|}{$11141(6781,77)$} & \multicolumn{5}{|c|}{0} \\
\hline CRP (ng/dL) & \multicolumn{2}{|c|}{$4,48(5,91)$} & \multicolumn{5}{|c|}{0} \\
\hline hsTnT (pg/mL) & \multicolumn{2}{|c|}{$34,81(20,32)$} & \multicolumn{5}{|c|}{27} \\
\hline NT-proBNP (pg/mL) & \multicolumn{2}{|c|}{$6654(10813,24)$} & \multicolumn{5}{|c|}{5} \\
\hline \multicolumn{8}{|c|}{ Blood Gas Analysis } \\
\hline pH (Mean SD) & \multicolumn{2}{|l|}{$\mathrm{PaO}_{2}$ (Mean SD) } & P/F (Mean SD) & \multicolumn{2}{|l|}{$\mathrm{PaCO}_{2}$ (Mean SD) } & \multicolumn{2}{|c|}{$\mathrm{HCO}_{3^{-}}$(Mean SD) } \\
\hline $7,38(1)(0,07)$ & \multicolumn{2}{|l|}{56,31 (1) $(13,93)$} & 229,68 (1) $(63,25)$ & \multicolumn{2}{|l|}{51,82 (1) $(12,07)$} & \multicolumn{2}{|c|}{31,02 (1) $(5,62)$} \\
\hline \multicolumn{8}{|l|}{ Chest imaging } \\
\hline Pattern & \multicolumn{2}{|l|}{ Normal } & Congestive & \multicolumn{2}{|l|}{ Bronchitis } & \multicolumn{2}{|c|}{ Emphysema } \\
\hline n. & \multicolumn{2}{|l|}{1} & 14 & \multicolumn{2}{|l|}{19} & \multicolumn{2}{|l|}{7} \\
\hline$\%$ & \multicolumn{2}{|l|}{2,43} & 34,14 & \multicolumn{2}{|l|}{46,34} & \multicolumn{2}{|c|}{17,07} \\
\hline \multicolumn{8}{|c|}{ Instrumental findings } \\
\hline Test & ECG abn & Diast Abn & W Disk & LVEF & PAPs & Spiro & $\mathrm{FEV}_{1}$ \\
\hline n. & 30 & 17 & 7 & $<55 \%: 8$ & $>35: 30$ & $32 \mathrm{ob} / 7 \mathrm{res}$ & $<30 \%: 15$ \\
\hline$\%$ & 73,17 & 54,83 & 17,07 & 19,51 & 73 & $78 / 17$ & 36,58 \\
\hline Mean SD & & & & $56,56(9,10)$ & $43(12,32)$ & & $34,24(12)$ \\
\hline $\mathrm{NE}$ & & 10 & & & 4 & & \\
\hline
\end{tabular}

Legenda: WBC: White Blood Cells; CRP: C Reactive Protein; TNT: high sensibility T troponin; NT-proBNP: n-terminal fragment of Brain Natriuretic Peptide; PaO ${ }_{2}$ : arterial Oxygen partial pressure (mmHg); P/F: the ratio between $\mathrm{PaO} 2$ and the fraction of inspired Oxygen; $\mathrm{PaCO}_{2}$ : arterial Carbon dioxide partial concentration (mmHg); $\mathrm{HCO}_{3}$ : bicarbonate ion (Meq/); ECG abn: Electrocardiographic abnormalities; Diast abn: abnormalities of the diastolic relaxation phase; W disk: wall diskinesis; LVEF: left ventricular ejection fraction (\%); PAPs: systolic pulmonary artery pressure (mmHg); Spiro: spirometry; Ob: obstructive pattern; Res: restrictive pattern; FEVl: Forced Expired Volume in the first second (\%predicted); NE: not evaluable 
in the pathophysiology of cardiovascular disorders and chronic obstructive pulmonary disease are conceivable. ${ }^{18}$ Surely the two diseases can be the effect of common lifestyles; in particular, exposure to cigarette smoke would lead, in addition to the wellknown effects of destruction of the lung parenchyma and vascular damage in general, to the production of an imbalance between oxidants and antioxidants at the pulmonary and systemic level, with maintenance of a chronic inflammatory state, which, in turn, would amplify the imbalance between oxidants and antioxidants. This would lead to lipoperoxidation of cell membranes, endothelial damage and proliferation of the vascular smooth muscle layer. ${ }^{19-22}$

These mechanisms would also underlie the processes leading to the development of pulmonary hypertension, although an increasing number of clinical and experimental observations indicate that COPD and pulmonary hypertension, although correlated, have a different natural history. ${ }^{23}$ Furthermore, some pathophysiological characteristics of COPD would have a direct effect on the function of the left ventricle, ${ }^{24}$ through mechanisms involving inflammation and oxidative stress, endothelial dysfunction and hypercoagulability, ${ }^{22}$ also induced by chronic hypoxemia, a common risk profile for coronary artery disease, and direct mechanical action conveyed by the condition of hyperinflation and ventricular interdependence. It should also be noted that in the field of research an increasing space is given to the use of "traditionally" cardiac markers in COPD, including troponins and natriuretic peptides. ${ }^{25}$

A certainly important aspect of cardiovascular comorbidities is their role in influencing the prognosis of the COPD patient affected by an exacerbation. It is widely recognized that the prognosis of the exacerbated patient largely depends on the severity of the exacerbation itself and of the underlying disease, hence the clinical importance of recognizing the signs of severity in the individual patient. ${ }^{1}$ However, acute cardiovascular events (heart failure, acute coronary syndrome) are found to have a greater severity and mortality in the patient with exacerbated COPD and, in turn, can determine a more severe course of the exacerbation itself, based on the interplay of inflammation factors mentioned above..$^{20,26}$

In our study the patients were of advanced severity, as evidenced by the high number of cases with $\mathrm{pH}$ below 7.35 and $\mathrm{PaO}_{2} / \mathrm{FiO}_{2}$ below 200, the high prevalence of high CRP values, the number of pneumonias, but also the high number of patients excluded from the study due to inability to perform spirometry due to persistence of dyspnea or respiratory failure.

In fact, nine patients $(21.9 \%)$ were hospitalized again for "COPD exacerbation" within three months of the index event, while, considering the period between the start of the study and the start of data processing (13.01.2016-15.05.2017) this event involved 14 cases $(34.1 \%)$. During this same period, 11 patients $(26.8 \%)$ died. In addition, patients included in the study had a mean length of stay in days of 7.2 (6.7); in 14 cases it lasted more than 7 days (34.1\%) (Table 4). On the one hand, these data confirm that the "exacerbation" event represents a negative prognostic factor in the natural history of COPD,${ }^{27}$ which should lead to a particular follow-up of these patients; ${ }^{20,28,29}$ on the other hand, it stimulates the search for factors potentially correlated with a worse outcome among exacerbated patients, including clinical and instrumental factors, but also, as expressed above, comorbidities.

As to our search for factors related to outcomes, given the low number of patients considered, the multivariate analysis did not allow us to associate the risk of death with any of the demographic, clinical, laboratory or instrumental variables considered; on the contrary, the presence of a history of heart failure was significantly correlated to the risk of new hospitalization within three months for COPD exacerbation (OR 7.14; 95\% CI 1.4-36; $\mathrm{p}<0.017$ ). Similarly, a hospital stay longer than seven days was significantly correlated with the presence of pneumonia (OR 18.28; 95\% CI; $\mathrm{p}$ $<0.008$ ); a low $\mathrm{pH}$ value at entry (OR $10.09 ; 95 \%$ CI 1.18-86.44; $\mathrm{p}<0.035$ ); and a measured FEV1 value less than $30 \%$ of predicted (OR 6.23; 95\% CI 0.98-39.5; $\mathrm{p}<0.052$ ). No significant correlations were observed between any of the variables considered and the risk of new hospitalization for exacerbated COPD throughout the longer study period (13.01.2016-15.05.2017) (Table 5) .

In the general population of COPD patients, the possibility of clustering some clinical features within homogeneous groups in terms of prognosis, therapeutic needs and evolution, has led to the

Table 4. Outcomes.

\begin{tabular}{lccccc} 
Outcomes & LOS & LOS $>7$ days & Adm $<3$ m & Adm $<16$ m & Deaths \\
n. & & 14 & 9 & 14 & 11 \\
$\%$ & $34,14 \%$ & $21,95 \%$ & 34,14 & $26,82 \%$ \\
\hline Mean (SD) & $7,24(6,74)$ & - & - & - & - \\
\hline
\end{tabular}

Legenda: LOS: Length of Hospital Stay (days); Adm < 3 m: new admission for COPD exacerbation within 3 months from the index event; Adm $<16$ m: new admission for COPD exacermation throughout the entire observation period (16 months)

Table 5. Multivariable analysis.

\begin{tabular}{lcccc} 
Variable & Adm $<3$ m & No Adm $<3$ m & OR $(95 \%$ CI $)$ & P \\
History of HF & 6 & 7 & $7,14(1,41-36)$ & 0,017 \\
No History of HF & 3 & 25 & OR $(95 \%$ CI $)$ & P \\
Variable & LOS $>7$ days & No LOS $>7$ days & $10,6(2,15-52,8)$ & 0,008 \\
\hline Pneumonia pres & 3 & 8 & $10,09(1,18-86,44)$ & 0,035 \\
Pneumonia abs & 6 & 24 & & \\
pH $<7.35$ & 6 & 6 & $6,3(1,52-26)$ & 0,002 \\
pH $>7.35$ & 8 & 21 & \\
FEVl $<30 \%$ pred & 9 & 6 & & \\
FEVl $>30 \%$ pred & 5 & 21 & & \\
\hline
\end{tabular}

Legenda: Adm < 3m: new admission for COPD exacerbation within 3 months from the index event; History of HF: History of Heart Failure: LOS > 7 days: Legnght of Hospital stay longer than 7 days; Pneupnia pres: pneumonia present at admission; Pneumonia abs: pneumonia absent at admission; FEV1: Forced Expired Volume in the first second (\% predicted). 
development of the concept of "phenotypes" of the disease quite recently. ${ }^{30}$ Although the declination of the various phenotypes varies between the different authors, ${ }^{31}$ a relatively simple classification on an operational level considers the presence of essentially three phenotypes: overlap asthma-COPD; frequent exacerbator; hyperinflated emphysematous. ${ }^{32}$ Of particular interest, for our purposes, is the "frequent exacerbator" patient, characterized by a clinical pattern of bronchitis-secretory type, activation of inflammation, high frequency of cardiovascular comorbidities and frequent exacerbations, and particular frequency of adverse events. In our series, patients with a history of heart failure were more likely to be hospitalized again for COPD exacerbation within three months of the index event, while the importance of general severity and inflammation is demonstrated by the role of FEV1, pH and pneumonia in influencing the length of hospital stay, as already published. ${ }^{33}$

Although existing documents do not address the problem of comorbidities in an organic way, ${ }^{1}$ a growing body of data suggests considering comorbidities as fundamental elements in the process of exacerbation and treating them individually. ${ }^{29,34-36}$

Some authors consider the presence of cardiovascular comobidity in these patients to be so important that it is not easy to distinguish which "exacerbation" to consider in each single event, whether cardiac or respiratory. ${ }^{32}$ This is particularly true if we consider that as many as 7 patients in our series, admitted to the ED with a diagnosis of "exacerbated COPD", were not found, at the spirometric survey carried out subsequently, to have obstructive disease. Since the purpose of the study was to characterize the entire population of patients who are hospitalized from the ER with this diagnosis, these patients were equally considered for data processing.

While thoracic ultrasound is increasingly established as a bedside technique for the diagnosis of the respiratory patient in the emergency department, it is possible that a relevant proportion of the overlaps between COPD and heart failure will be detected by this method. ${ }^{37}$ It should also be noted that while thoracic ultrasound is very sensitive in confirming the presence of pulmonary congestion in the COPD patient, it cannot detect an obstructive airway condition in the patient with heart failure. ${ }^{38,39}$

The present study has many limitations, first of all the low sample size and the monocentric character. As already mentioned, this fact prevents any general conclusions from being drawn from the results. However, the interest of the researchers was above all to highlight a substantial heterogeneity in the population of patients admitted to the ED with a diagnosis of exacerbated COPD, referring the possibility of demonstrating its clinical and operative significance to much larger and multicentre studies. It should also be noted that some of our results confirm previous data produced in more numerous studies. Furthermore, the small number of the sample also depended on the loss of many patients at enrollment as they were too serious, and this could have induced a selection bias. However, the patients in our case series were also very serious for the above reasons and it is therefore conceivable that the inclusion of a greater number of patients could certainly have expanded, but would hardly contradict the result of our study. In our study the possible presence of patients with asthma-COPD overlap syndrome was not considered. However, it is a phenotype still being defined, ${ }^{40,41}$ moreover, with the limitations of this data, all the patients in our series have carried out the spirometric examination during maximal bronchodilating therapy and none of them reported clinical characteristics in their history attributable to an asthmatic condition. The study protocol did not include a defined therapeutic scheme and the therapeutic choices were left to the physician in charge. All patients, however, received antibiotic, bronchodilating and steroid therapy according to existing evidence (GOLD), with further interventions related to specific and individual needs; we therefore believe that this factor did not significantly influence the results.

In conclusion, our study demonstrates that the population of patients who refer to the Emergency Department diagnosed with "exacerbated COPD" represent a very heterogeneous population of patients, characterized by a high prevalence of comorbidities, in particular cardiovascular and that these, together with the clinical severity and the presence of inflammatory-infectious causes of the exacerbation can significantly influence the patient's prognosis in the short to medium term. It is desirable that more numerous and multicenter studies allow the identification of specific risk factors that facilitate differentiated management and a particular followup to those patients who are at greater risk of adverse events in the short-medium term, ultimately leading to a reduction in morbidity and mortality of the disease and a more effective allocation of resources.

\section{References}

1. Global Initiative for Chronic Obstructive Lung Disease: Global Strategy for the diagnosis, management and prevention on Chronic Obstructive Pulmonary Disease, 2020 Report.

2. Agusti A, Calverley PM, Celli B, et al. Characterisation of COPD heterogeneity in the ECLIPSE cohort. Respir Res 2010;11:122.

3. Miravitlles M, Calle M, Soler-Cataluña JJ. Clinical phenotypes of COPD: identification, definition and implications for guidelines. Arch Bronconeumol 2012;48:86-98.

4. Pinto LM, Alghamdi M, Benedetti A, et al. Derivation and validation of clinical phenotypes for COPD: a systematic review. Respir Res 2015;16:50.

5. Cavaillès A, Brinchault-Rabin G, Dixmier A, et al. Comorbidities of COPD. Eur Respir Rev 2013;22:454-75.

6. Smith MC, Wrobel JP. Epidemiology and clinical impact of major comorbidities in patients with COPD. Int J Chron Obstruct Pulmon Dis 2014;9:871-88.

7. Burgel PR, Paillasseur JL, Caillaud D, et al. Initiatives BPCO Scientific Committee. Clinical COPD phenotypes: a novel approach using principal component and cluster analyses. Eur Respir J 2010;36:531-9.

8. Vanfleteren LE, Spruit MA, Groenen M, et al. Clusters of comorbidities based on validated objective measurements and systemic inflammation in patients with chronic obstructive pulmonary disease. Am J Respir Crit Care Med 2013;187:72835.

9. Garcia-Aymerich J, Gómez FP, Benet M, et al. Identification and prospective validation of clinically relevant chronic obstructive pulmonary disease (COPD) subtypes. Thorax 2011;66:430-7.

10. Miller MR, Hankinson J, Brusasco V, et al. Standardisation of spirometry Eur Respir J 2005;26:319-38.

11. Pellegrino R, Viegi G, Brusasco V, et al. Interpretative strategies for lung function tests Eur Respir J 2005;26:948-68.

12. Official Statement of the European Respiratory Society ( No authors listed). Standardized Lung function testing. Eur Respir J Suppl. 1993 Mar;16:1-100.

13. Rodriguez Roisin R. Towards a consensus definition for COPD exacerbation. Chest 2000;117:398s-401s. 
14. Lou P, Zhu Y, Chen P et al. Vulnerability, beliefs, treatment and economic burden of chronic obstructive pulmonary disease in rural areas in China: a cross sectional study BMC Public Health 2012;12:287.

15. Ko FW, Chan KP, Hui DS, et al. Acute exacerbation of COPD. Respirology 2016;21:1152-65.

16. Decramer M, Iassens W. Chronic pulmonary disease and comorbidities. Lancet Respir Med 2013;1:73-83.

17. Brown JP, Martinez CH. Chronic obstructive pulmonary disease comorbidities. Current Opinion Pulm Med 2016;22:11318.

18. Hawkins NM, Virani S, Ceconi C. Heart failure and chronic obstructive pulmonary disease: the challenges facing physicians and health services. Eur Heart J 2013;34:2795-803.

19. Bernardo I, Bozinovski S, Vlahos R. Targeting oxidant-dependent mechanisms for the treatment of COPD and its comorbidities. Pharmacol Ther 2015;155:60-79.

20. Morgan AD, Zakeri R, Quint JK. Defining the relationship between COPD and CVD: What are the implications for clinical practice? Ther Adv Respir Dis 2018;12:1-16.

21. Rabe KF, Hurst JR, Suissa S. Cardiovascular disease and COPD: dangerous liaisons? Eur Respir Rev 2018;27:180057.

22. Brassington K, Selemidis S, Brozinovski S, Vlahos R. New frontiers in the treatment of comorbid cardiovascular disease in chronic obstructive pulmonary disease. Clin Sci 2019;133:885-904.

23. Neder JA, Rocha A, Alencar MCN, et al. Current Challenges in managing comorbid Heart failure and COPD. Expert Rev Cardiovasc Ther 2018;16:653-73.

24. Portillo K, Abad-Capa J, Ruiz-Manzano J. Chronic Obstructive Pulmonary Disease and Left Ventricle. Arch Bronconeumol 2015;51:227-34.

25. Buchan A, Bennet R, Coad A, et al. The role of cardiac biomarkers for predicting left ventricle disfunction and cardiovascular mortality in acute exacerbations of COPD. Open Heart 2015;2:e000052.

26. Almagro P, Boixeda R, Diez-Manglano J, et al. Insights into Chronic Obstructive Pulmonary Disease as Critical Risk Factor for Cardiovascular Disease. Int J Chronic Obst Pulmonary Disease 2020;15:755-64.

27. Rowe BH, Bhutani M, Stickland MK, Cydulka R. Assessement and management of Chronic obstructive pulmonary disease in the emergency department and beyond. Expert Rev Respir
Med 2011;5:549-59.

28. Andre S, Conde B, HìFragoso E et al. COPD and Cardiovascular Disease. Pulmonol 2019;25:168-76.

29. Canepa M, Franssen FME, Olschewski H, et al. Diagnostic and Therapeutic gaps in patients With heart failure and chronic obstructive pulmonary disease. J Am Coll Cardiol HF 2019;7:823-33.

30. Zhou A, Zhou Z, Zhao Y, Chen P. The recent advances of Phenotypes in acute exacerbations of COPD. Int $\mathrm{J}$ COPD 2017; 12:1009-101.

31. Lange P, Halpin DM, O'Donnell DE, MacNee W. Diagnosis, assessment, and phenotyping of COPD: beyond $\mathrm{FEV}_{1}$. Int $\mathrm{J}$ Chron Obstruct Pulmon Dis 2016;11:3-12.

32. Miravittles M, Calle M, Soler-Cataluna JJ. Clinical phenotypes of COPD: identification, definiton and implications for guidelines. Arch Bronconeumol 2012;48:86-9.

33. Santos S, Marin A, Serra Battles J, et al. Treatment of patients with COPD and recurrent exacerbations: the role of infection and inflammation. Int J COPD 2016;11:515-25.

34. Van Fleteren LEGS, Private MA, Wouters EFM, Franssen FME. Management of chronic obstructive pulmonary disease beyond the lungs. Lancet Respir Med 2016;4:911-24.

35. Hillas G, Periklos F, Tsiligianni I, Yzanakis N. Managing comorbidities in COPD. Int J COPD 2015;10:95-109.

36. Lipworth B, Wedzicha J, Devereux G, et al. Beta-blockers in COPD: time for reappraisal Eur Respir J 2016;48:600-3.

37. Zanobetti M, Scorpiniti M, Gigli C, et al. Point-of-care ultrasonography for evaluation of Acute dyspnea in the Emergency Department. Chest 2017;151:1295-1301.

38. Sriram KB, Singh M. Lung ultrasound B-lines in exacerbations of chronic obstructive Pulmonary disease. Intern Med J 2017; 47:324-27.

39. Corradi F, Brusasco C, Brusasco V. When, Where and How to use lung ultrasonography in Patients with chronic obstructive pulmonary disease. Arch Bronconeumol 2017;53:229-30.

40. Nielsen M, Boslev-Barnes C, Ulrik CS. Clinical characteristics of the asma-COPD overlap syndrome- a systematic review. Int J COPD 2015;10:1443-54.

41. Ding B, Enstone A. Asthma and chronic obstructive pulmonary disease overlap syndrome-ACOS: structured literature review and physicians insights. Expert Rev Res Med 2016;10: 363-71. 